\title{
Personality, cognitive skills and life outcomes: evidence from the Polish follow-up study to PIAAC
}

\author{
Marta Palczyńska ${ }^{1,2^{*}}$ and Karolina Świst ${ }^{1}$
}

\author{
*Correspondence: \\ m.palczynska@ibe.edu.pl \\ ${ }^{1}$ Educational Research \\ Institute, Warsaw, Poland \\ Full list of author information \\ is available at the end of the \\ article
}

\begin{abstract}
Background: There is a growing literature providing evidence on the importance of non-cognitive skills for life outcomes. However, to date there is limited evidence on the gains from incorporating such measures into large-scale competence surveys.

Methods: We investigate the relationship between personality traits and eight important life outcomes: educational attainment, labour market participation, employability, wages, job satisfaction, health, trust and life satisfaction measured in the Polish followup study to PIAAC. The study assesses two short scales: the Big Five Inventory and Grit. First, we compare explanatory power of personality traits to that of cognitive skills measured by PIAAC. Second, an incremental validity of Grit after controlling for the Big Five dimensions is assessed.
\end{abstract}

Results: The analyses show that differences in personality traits are important in explaining differences in life outcomes. Educational attainment is more strongly related to cognitive skills, while for wages, the explanatory power of personality and cognitive skills is similar. For most of the subjective outcomes, the Big Five traits outperform cognitive skills in predictive power. Conscientiousness is positively related to most of the outcomes analysed while Neuroticism has a negative relationship. After controlling for sociodemographic characteristics and cognitive skills, Big Five traits add explanatory power to all models except for employability. Grit explains some additional variation in educational attainment and in a number of subjective outcomes: health, trust, job and life satisfaction, even after adjusting for the effects of cognitive skills and Big Five traits.

Conclusions: Given the potential benefits and relatively small burden on respondents in terms of required time it seems advisable to incorporate measures of personality traits into competence surveys as they contribute to explaining the variability in policyrelevant outcomes. The use of the Big Five Inventory seems preferable to Grit when a broad range of life outcomes is of interest, as the former covers multiple aspects of personality. However, using both scales offers an improvement in explanatory power.

Keywords: PIAAC, Cognitive skills, Personality, Grit, Earnings, Health, Well-being, Perseverance of effort, Consistency of interest 


\section{Background}

There is a consensus that cognitive skills have important effects on economic and social outcomes. The evidence is ample, both in national and cross-national data. For over two decades comparable international surveys on cognitive skills have been conducted, ${ }^{1}$ the most recent being the Programme for the International Assessment of Adult Competencies (PIAAC) coordinated by the OECD. The programme confirmed the importance of cognitive skills for economic and social outcomes in the participating countries (da Costa et al. 2014; OECD 2013a; OECD 2016).

However, it has also been recognised that cognitive skills as measured by achievement tests are not the only determinants of a successful life. There is a growing literature providing evidence on the importance of non-cognitive skills for life outcomes (for reviews, see Almlund et al. 2011; Borghans et al. 2008).

The striking causal evidence on the predictive power of non-cognitive skills has come from the Perry Preschool Program. This experimental intervention, targeted at disadvantaged 3-year-old children, included weekly home visits to enrich children-parent interactions and preschool education with a curriculum aimed at fostering children's cognitive and socio-emotional skills. The programme lasted 2 years and both treatment and control groups were followed through to age 40 (Heckman et al. 2010a). The programme did not boost IQ in the long run but did produce significant treatment effects for educational and economic outcomes and crime (Heckman et al. 2010b). Heckman et al. (2013) show that, to a large extent, the effectiveness of the programme can actually be attributed to persistent changes in non-cognitive skills.

The general educational development (GED) programme in the USA provides further evidence of the importance of non-cognitive skills for educational and economic outcomes. This programme offers high school dropouts the possibility to formally certify that their cognitive skills are equivalent to those of high school graduates. Despite the fact that their cognitive skills are similar, they nevertheless perform worse in the labour market than high school graduates without further college education. Heckman and Kautz (2012) show that the non-cognitive skills of GED recipients are closer to high school dropouts than to graduates, which results in the differences in the labour market performance.

Although it is difficult to draw a sharp line between cognitive and non-cognitive skills (Almlund et al. 2011), the term "non-cognitive skills" is used by economists for a wide range of traits which are believed to be distinct from skills measured by IQ tests and achievement tests, such as personality, motivation or interests. Special attention has been paid to the concept of personality. One of the most widely used frameworks for describing human personality is the Big Five model. It identifies five dimensions of personality: Openness to experience, Conscientiousness, Extraversion, Agreeableness, and Neuroticism (Costa and McCrae 1985; John and Srivastava 1999; McCrae and Costa 1999).

A large volume of empirical literature studies the predictive power of personality traits. These studies show that personality, and especially traits connected to

${ }^{1}$ These included studies on school-aged children: PISA, TIMSS, PIRLS; and on the adult population: IALS, ALL, PIAAC. 
Conscientiousness and Neuroticism, predicts a wide range of important life outcomes. Conscientiousness is positively related to job performance, training proficiency and personnel data (such as salary or promotions), while Openness and Extraversion predict training proficiency (Barrick and Mount 1991). Various studies have shown that there is a wage penalty for Agreeableness and Neuroticism (Mueller and Plug 2006; Nyhus and Pons 2005; O'Connell and Sheikh 2011). Studies on US samples note a positive association between Openness and wages (Mueller and Plug 2006; O'Connell and Sheikh 2011). The importance of the traits relating to Neuroticism/Emotional stability, such as selfesteem and locus of control, for the wage setting has been confirmed by Drago (2011) and Heineck and Anger (2010). Educational attainment is best predicted by Openness to Experience (Goldberg et al. 1998; O'Connell and Sheikh 2011; Van Eijck and de Graaf 2004). The first two studies also report a positive but much weaker association of Conscientiousness with years of education. Openness to Experience, also called Intellect, has facets of Ideas and Fantasy which are often related to measured intelligence (Almlund et al. 2011). Hampson et al. (2007) find that Extraversion, Agreeableness and Conscientiousness, measured during childhood, predict self-rated health and healthy behaviours in midlife. Part of this effect is indirect through educational attainment. The same traits have positive effects on longevity (Roberts et al. 2007). The latter meta-analysis also shows that all personality traits are stronger predictors of mortality than socio-economic status and are comparable to IQ. Overall, among the Big Five traits, Conscientiousness is the strongest predictor of health outcomes. The evidence on the relationship between personality and generalised trust is mixed. Only the result on the positive link between Agreeableness and trust emerges consistently between many studies (Anderson 2010; Dohmen et al. 2008; Freitag and Bauer 2016). Additionally, some studies find that Openness is also positively related to trust (Dohmen et al. 2008; Freitag and Bauer 2016). Dohmen et al. (2008) show that Neuroticism and Conscientiousness are negatively linked to trust. By contrast, Freitag and Bauer (2016) find that Conscientiousness relates positively to trust. Personality can also explain individual differences in life satisfaction. A meta-analysis indicates that Neuroticism and Extraversion are the strongest predictors of subjective well-being among the Big Five traits (DeNeve and Cooper 1998). More recently, using the British Cohort Study 1970, Prevoo and Ter Weel (2015) show that Extraversion in childhood is the strongest predictor of life satisfaction around 30 years later, while Neuroticism does not have a significant influence. When analysing workers' well-being, a meta-analysis by Judge et al. (2002) indicates that extraverts are more likely to be satisfied with their work, while neurotic individuals are less likely to be satisfied. Using German PIAAC longitudinal data, Rammstedt et al. (2017) show that after controlling competences, Big Five traits incrementally predict life satisfaction and health, and to a lesser extent income, educational attainment and employability.

In sum, Big Five traits play an important role in predicting a wide range of economic and social outcomes. While most of the literature examines the effect in the United States and Western Europe, the study by Cunningham et al. (2016) is an exception. Using the Peruvian National Skills and Labor Market Survey, they find that Openness and Emotional Stability are related to wages, and that an aggregate of these two-plasticity-is related to employment. There is also a negative link between traits connected to Agreeableness and wages. Additionally, the study includes one of the Grit subscales: 
Perseverance of effort. They show that it is positively correlated with employment when controlling for Conscientiousness.

Although the Big Five taxonomy is probably the most widely used personality framework, new research on personality traits is emerging nowadays. The trait termed Grit deserves special attention, as a relatively new construct that quickly attracted attention and started to be collected in international surveys such as the Skills Towards Employment and Productivity (STEP) and was even included in the 2017 edition of the National Assessment of Educational Progress (NAEP) in the US (Bertling 2016). It is not part of the Big Five model but can be related to it. Grit is defined as perseverance and passion for long-term goals. People with a high Grit level sustain their interest and effort in an activity, despite challenges, failures and a lack of positive feedback (Duckworth et al. 2007; Duckworth and Quinn 2009). Grit is a similar construct to one of the Big Five factors-Conscientiousness-which is defined as a "socially prescribed impulse control that facilitates task- and goal-related behaviour" (John and Srivastava 1999). It consists of such traits as being careful, thorough, responsible and organised. Additionally, and just like Grit, it includes volitional traits such as being hardworking, achievement-oriented and persevering (Barrick and Mount 1991). However, it is argued that Grit and Conscientiousness are conceptually different as Grit emphasises stamina: being able to sustain effort and interest in projects which take a lot of time to complete (Duckworth et al. 2007). Nevertheless, both constructs correlate strongly. The results vary from 0.44 (Ivcevic and Brackett 2014) to 0.64 and 0.74 for the Consistency of interest and Perseverance of effort subscales respectively (Duckworth and Quinn 2009). A twin study by Rimfeld et al. (2016) shows that Grit is similar to other psychological traits (for example Big Five traits) in its nature-it shows genetic influence (heritability of Perseverance of effort $=37 \%$ and of Consistency of interest $=20 \%$ ) and no shared environmental influence. They conclude that perseverance of effort and Conscientiousness are both phenotypically and genetically correlated.

Although Grit has a much shorter tradition than the Big Five model, the body of evidence on its predictive power is growing. The largest body of literature concerns the relationship between the level of Grit and educational attainment. Eskreis-Winkler et al. (2014) show that grittier individuals are more likely to graduate from high school, and that the relationship holds also when controlling for academic Conscientiousness. Moreover, individuals with a higher level of Grit have smaller chances of dropping out of education or the labour market (becoming NEETs - not in education, employment or training) at age 18-20 (Mendolia and Walker 2014). According to Duckworth and Quinn (2009), children with a high Grit level are more likely to win in spelling competitions and grittier adolescents obtain higher GPAs (Grade Point Average). However, Bazelais et al. (2016) analysed a sample of college students to find that Grit is an insignificant predictor of academic achievement, when controlled for the prior academic performance. A recent meta-analysis by Credé et al. (2017) finds that Grit is related to academic performance and retention but its facets differ in terms of the strength of this relationship: the Perseverance of effort facet is related more strongly to all academic performance criteria than the Consistency of interest facet.

Grittier individuals also tend to have more stable life outcomes: they make less career changes (when controlled for other personality traits and age), drop out less frequently 
during training, and have longer-lasting marriages (Eskreis-Winkler et al. 2014; Duckworth and Quinn 2009). The positive effect of Grit on academic and labour performance exists both in individualistic and collective cultures (Datu et al. 2016; Suzuki et al. 2015). Although most of the researchers focus on the positive influence of Grit, Lucas et al. (2015) show in their experiment that too much Grit can be harmful. Grittier participants are less likely to quit activities even when they fail or the activities cost them too much.

There is mixed empirical evidence on the incremental validity of Grit. When controlling for Conscientiousness and the other Big Five traits, Duckworth and Quinn (2009) find that grittier individuals attain higher levels of education than other individuals of the same age. However, Dumfart and Neubauer (2016) find no incremental contribution of Grit when analysing the combined impact of intelligence and Conscientiousness on secondary school achievement (measured by GPA, science and languages). Rimfeld et al. (2016), in their twin study, find that personality traits (mainly Conscientiousness) predict about 6\% of variance in GCSE (General Certificate of Secondary Education) grades but Grit incremental validity is low. Credé et al. (2017) find that overall Grit does not explain additional variance in academic performance (measured by GPAs in high school, college, and individual grades) after controlling for Conscientiousness, but that Perseverance of effort facet explains a substantial incremental variance in all three measures of academic performance even after controlling for Conscientiousness. Their results suggest that the two Grit facets should be analysed separately.

Almlund et al. (2011) summarise why personality should not be ignored in the research of life outcomes. First, personality traits have comparable predictive power for important outcomes to measures of cognition. Moreover, very often performance in achievement tests depends not only on cognition but also on personality. The authors also highlight the relevance of personality traits for policy interventions, as these traits are more malleable than cognition; hence interventions aimed at boosting non-cognitive skills can be a way of addressing social problems.

Culture may influence personality-outcome relationships. As Henrich et al. (2010) argue, the results of Western empirical studies can be generalised to other contexts only with great caution. One of the established dimensions of culture is individualism/collectivism (Hofstede 1980). In the individualistic context, there is an emphasis on personal autonomy and self-fulfilment, whereas collectivist cultures attach more value to interpersonal harmony and common group goals (Inglehart 2006). There is some evidence that collectivist culture moderates the personality-economic outcomes relationship (Grijalva and Newman 2015). Further, Datu et al. (2016) argue that the Consistency of interest facet of Grit is less relevant in collectivist cultures. On the other hand, a few studies on personality-economic outcomes relationships in collectivist cultures have replicated earlier results from the US and Western Europe (Cunningham et al. 2016; Suzuki et al. 2015). More research in diverse cultural contexts is needed to assess the impact of culture on the relationships under investigation.

One of the challenges in personality assessment in large-scale studies is the measurement of personality traits. Unlike standardised achievement tests, personality tests are based on self-reported measures, which are less objective. The only international survey that collects information, both on cognitive and non-cognitive skills, is the STEP study conducted in developing countries. The preliminary findings show that more 
conscientious, emotionally stable and grittier (determined) workers find their first job faster. Likewise, non-cognitive skills are associated with higher wages (World Bank 2014).

However, we still lack an international study that systematically evaluates the combined impact of cognitive and non-cognitive skills in developed countries. While the first cycle of PIAAC made an assessment across three domains-literacy, numeracy and problem solving in technology-rich environments-no non-cognitive measures are available. The second cycle of PIAAC is planned for 2018-2023 and will be an opportunity to build on experience from the previous cycle. One of the possible changes might be to include new areas of assessment, particularly non-cognitive skills.

The objective of this paper is to assess the analytical importance of personality measures compared to competences measured in PIAAC and to compare the criterion validity of the Big Five and Grit constructs in a large representative sample of adults in Poland. In a related study, Rammstedt et al. (2017) used the data from the German PIAAC longitudinal study to assess the predictive power of Big Five traits. They show that, after accounting for competencies, personality explains incremental variance in educational attainment, employment status, income, life satisfaction, and health. In our article, we extend this research by comparing criterion validity of the two popular scales: Big Five and Grit, also taking into account the additional life outcomes of labour force participation and job satisfaction. Moreover, we investigate the incremental validity of Grit after adjusting for Big Five dimensions. To the best of our knowledge, this is the first article to provide evidence on the relationship between personality and important life outcomes in the Polish adult population.

The paper is organised as follows. In the next section, we describe the data set and the measures used. We then investigate the relationship between cognitive and non-cognitive skills and life outcomes. This is followed by the discussion of the incremental validity of Grit. The last section concludes.

\section{Methods}

\section{Research design and sample}

We analyse the data from the Polish Follow-up Study to the Programme for International Assessment of Adult Competencies (postPIAAC). The main goals of the study were to gather longitudinal information on PIAAC respondents in Poland and to collect additional background information not available in the international study. The background questionnaire $(\mathrm{BQ})$ of postPIAAC is based on the PIAAC international questionnaire with many additional questions and some modifications. The methodology of collecting labour market outcomes was not changed. Regarding other outcomes, a question on life satisfaction was added and the question on political efficacy was removed, leaving the rest of the social indicators unchanged (health and trust). The BQ was administered as a computer-assisted personal interview (CAPI). The study included parts with direct assessment, both on computer (a working memory test and a basic ICT skills test) and on paper (a coding speed test, a Big Five personality test and a self-assessment of skills). The Grit test was part of the BQ. The analysis is based on postPIAAC data combined with the proficiency estimates from PIAAC. The interval between the interviews for an 
individual respondent is from 2.5 to 3.5 years. The analysis thus assumes that respondents' cognitive skills have not changed significantly between the two waves.

The target population for PIAAC included all non-institutionalised individuals aged 16-65, residing in Poland during the period of data collection in 2011-2012. The target population of postPIAAC were PIAAC respondents who lived in Poland during the fieldwork conducted between October 2014 and February 2015 (aged 18-69 at that time). PIAAC respondents who had either died or emigrated between the interviewers' visits were classified as ineligible in postPIAAC. The weighting process of the postPIAAC sample was based on PIAAC guidelines (OECD 2013b). The final weight in PIAAC was taken as the person base weight in postPIAAC. The next step involved correcting for non-response in order to reduce potential bias arising from differences between respondents and non-respondents. Using a classification tree methodology, adjustment cells were constructed which were homogeneous with respect to the response rate. The calibration referred to the population estimates produced by PIAAC with respect to age, gender and proficiency score. The weighting process ensured that the average PIAAC results are replicated between the original and postPIAAC sample with regard to standard characteristics such as gender, age or educational attainment. Additionally, replication weights (paired jackknife) were computed in order to facilitate the estimation of variance.

Of the initial 9366 respondents in PIAAC, 5224 completed postPIAAC interviews in 2014/2015. After selecting individuals with valid answers to the relevant outcome questions, we kept a working sample of 4454 for the analysis of life outcomes. The working sample for job quality outcomes was further reduced to 2507 and 2059 respectively for job satisfaction and wages (only dependent workers).

\section{Measures}

\section{Personality}

The study includes two self-reporting scales: the Big Five Inventory-Short (BFI-S) (Gerlitz and Schupp 2005; John et al. 1991) and the short eight-item Grit scale (Grit-S) (Duckworth and Quinn 2009). BFI-S includes 3 items per dimension with answers on a seven-point Likert type scale (1-“disagree completely" to 7-"agree completely"). Grit scale is answered on a five-point scale ranging from 1-"not like me at all" to 5-"very much like me". Palczyńska and Świst (2016) perform an item-level analysis of BFI-S and Grit-S scales using both Classical Test Theory and Item Response Theory techniques. They show that most of the Big Five items discriminate well between people possessing a high and a low level of a given trait, though the reverse-worded items perform weaker. The reliability of scales is moderate but satisfactory given their length (three items per each subscale) and improves after removing problematic items. ${ }^{2}$ All Grit items, except one, function well psychometrically and its subscales have comparable reliability (standardised Cronbach's alpha values $0.65-0.67$ ).

\footnotetext{
2 The standardised Cronbach's alpha values range from 0.36 for Extraversion to 0.61 for Conscientiousness and after removing negative items they range from 0.45 for Agreeableness to 0.66 for Conscientiousness.
} 
The BFI-S theoretical five-factor structure was not replicated in the Polish adult population sample. ${ }^{3}$ However, literature suggests that reverse-worded items form a separate factor (DiStefano and Motl 2006). A six-factor oblique model with an additional factor loading reverse-worded items provides satisfactory fit with the data (RMSEA $=0.077$, $\mathrm{TLI}=0.860, \mathrm{CFI}=0.905)$. In case of Grit-S, the second-order theoretical structure does not hold in our sample as it is not identified. A two-factor model supported by the recent literature (Credé et al. 2017; Midkiff et al. 2017) provides good fit with the data (RMSEA: 0.059, CFI: 0.964, TLI: 0.947). We use factor score from the six-factor oblique model for Big Five and from the two-factor model for Grit-S in multivariate analyses presented in the paper. We generated factor scores using the regression method for both scales. As researchers have often used Grit-S as a single factor (Duckworth et al. 2011; EskreisWinkler et al. 2014), and computing overall Grit from all the items is recommended by Duckworth et al. (2007), we also compare our results from the two-factor model to the unidimensional model. However, one has to note that the unidimensional model has poorer fit to the data (RMSEA: 0.135, CFI: 0.804, TLI: 0.726).

The scores for BFI-S and Grit-S subscales are standardised with a mean of zero and a standard deviation of one.

\section{Cognitive skills}

PIAAC measures basic information processing skills: literacy and numeracy. They represent acquired knowledge, sometimes called crystallized intelligence. Literacy is defined as "the ability to understand, evaluate, use and engage with written texts to participate in society, to achieve one's goals, and to develop one's knowledge and potential" (OECD 2013a). Numeracy refers to "the ability to access, use, interpret and communicate mathematical information and ideas in order to engage in and manage the mathematical demands of a range of situations in adult life." (OECD 2013a) Both skill domains are measured on a 500-point scale. For analytical purposes, we standardise scores in the subsequent analyses to have a mean of zero and a standard deviation of one. Although intended to measure different skills, PIAAC literacy and numeracy scales are strongly correlated ( 0.85 in our sample). The subsequent analyses use numeracy measure but the results for literacy are not qualitatively different. ${ }^{4}$

PIAAC uses multiple imputations (plausible values-PVs) to increase the accuracy of the cognitive measures (for details see OECD 2013b). Ten PVs are drawn for each respondent per domain. We ran all the regression analyses separately for each of the ten PVs and report the average results with the imputation error added to the variance estimator. ${ }^{5}$

\section{Life outcomes}

The present study analyses eight important life outcomes: educational attainment, labour force participation, employability, wages, job satisfaction, health, trust and life

\footnotetext{
3 The five-factor orthogonal model yields the following fit statistics: RMSEA $=0.199, \mathrm{TLI}=0.450, \mathrm{CFI}=0.542$, whereas the five-factor oblique model: $\mathrm{RMSEA}=0.127, \mathrm{TLI}=0.625, \mathrm{CFI}=0.725$

4 Results for literacy are presented in Additional file 1.

${ }^{5}$ Stata command repest is used.
} 
satisfaction measured in postPIAAC. For each outcome we provide the analogous variable from PIAAC in brackets.

Educational attainment: Educational attainment was measured in years based on the highest level of education reported by the respondent [B_Q01aPL].

Labour force status: Labour force status was assessed using a series of questions based on ILO (International Labour Organization) methodology. Labour force participation means being active on the labour market (employed or unemployed). Employability was assessed among active individuals [C_D05].

Wages: Respondents were allowed to report wages for different time intervals. The answers were recalculated into hourly wages based on hours worked per week. This outcome is analysed among dependent workers. Logarithm of wages is used throughout the analysis [EARNHR].

Job satisfaction: Respondents assessed their job satisfaction on a five-point scale from "extremely satisfied" to "extremely dissatisfied" [D_Q14].

Health: Respondents rated their general health on a five-point scale from "excellent" to "poor" [I_Q08].

Trust: The indicator of trust is an average of two items on social trust: "There are only a few people you can trust completely" and "If you are not careful, other people will take advantage of you" [I_Q07a, I_Q07b]. The respondents could agree with the statements on a five-point scale ranging from "strongly agree" to "strongly disagree".

Life satisfaction: Current life satisfaction was measured with one item on a seven-point scale ranging from "extremely satisfied" to "extremely dissatisfied" [No question on life satisfaction in PIAAC].

Descriptive statistics of life outcomes can be found in Table 9 in Appendix. Categories of ordinal variables with a frequency below $5 \%$ were merged with the neighbouring category.

\section{Control variables}

In all the analyses, besides the main variables of interest: personality and cognitive skills, we included controls for demographic and socio-economic characteristics. Specifically, we controlled for age, age squared, gender and years of education. ${ }^{6}$ For some of the outcomes, additional controls were included. In case of educational attainment we included father's education and mother's education in the model, in the analysis of social outcomes we added employment status and in the analysis of labour market outcomes the occupation (using 9 groups from ISCO classification). Army workers $(\mathrm{ISCO}=0$ ) were excluded from the analysis.

\section{Methods}

We used ordinary least squares regression to analyse all the outcomes as comparability over models is very important for the purposes of this study. First, this approach allows us to compare the effect estimates between analyses of different outcomes. Second, it overcomes the problem of incomparable coefficients in models with different

\footnotetext{
${ }^{6}$ Except for the analysis of educational attainment.
} 
Table 1 Educational attainment and cognitive and non-cognitive skills

\begin{tabular}{|c|c|c|c|c|c|c|c|}
\hline & (1) & (2) & (3) & (4) & (5) & (6) & (7) \\
\hline Numeracy & & $0.323^{* * *}$ & & & $0.315^{* * *}$ & $0.319^{* * *}$ & $0.310^{* * *}$ \\
\hline Conscientiousness & & & -0.043 & & -0.007 & & -0.061 \\
\hline Extraversion & & & $-0.086^{* *}$ & & $-0.060^{*}$ & & $-0.059^{*}$ \\
\hline Agreeableness & & & -0.080 & & $-0.087^{*}$ & & -0.066 \\
\hline Openness & & & $0.172^{* * *}$ & & $0.141^{* * *}$ & & $0.144^{* * *}$ \\
\hline Neuroticism & & & $-0.051^{* *}$ & & $-0.037^{*}$ & & -0.019 \\
\hline Perseverance of effort & & & & -0.002 & & 0.027 & 0.043 \\
\hline Consistency of interest & & & & $0.107^{* * *}$ & & $0.071^{* *}$ & $0.072^{* * *}$ \\
\hline Observations & 4355 & 4355 & 4355 & 4355 & 4355 & 4355 & 4355 \\
\hline$R^{2}$ & 0.296 & 0.385 & 0.310 & 0.306 & 0.394 & 0.393 & 0.403 \\
\hline
\end{tabular}

${ }^{*} p<0.05,{ }^{* *} p<0.01,{ }^{* * *} p<0.001$ Control variables: age, age squared, gender, father's education and mother's education. Dependent variable, numeracy and non-cognitive skills are standardised. Full estimation results are presented in Additional file 3: Table S1

independent variables (Mood 2010). We performed sensitivity analyses using non-linear models when appropriate and the results are qualitatively similar to the linear models in terms of the sign and the significance of the relationship between non-cognitive skills and life outcomes. ${ }^{7}$

To assess the incremental validity of Grit after controlling for the Big Five we compare nested specifications checking whether Perseverance of effort and Consistency of interest explain incremental variance and whether the relationship of Big Five traits with the outcomes changes after adjusting for Grit subscales.

The complex survey design and sampling weights have been accounted for in the estimations of the parameters in the regression analysis.

\section{Results}

In order to investigate the relationships between the personality scales and the outcomes and compare them to the impact of cognitive skills we estimate seven models for each outcome. The first one includes control variables only and serves as a benchmark. The next three specifications separately consider the associations between the outcome and numeracy, Big Five and Grit. Specifications 5 and 6 include numeracy and Big Five or Grit respectively, while the last column shows the incremental validity of Grit when controls are included for numeracy and the Big Five. The first part of this section focuses on specifications 1-6 while the results on the incremental value of Grit for the prediction of life outcomes are covered in a separate subsection.

\section{Educational attainment}

The variance explained by cognitive skills rivals that explained by measured personality traits (Table 1, specifications 2-4). Including personality traits (either Big Five or Grit) in regressions with cognitive skills explains around $1 \%$ of the additional variance (specifications 5-6). Openness is associated with higher levels of education while Extraversion,

\footnotetext{
${ }^{7}$ Results are presented in Additional file 2.
} 
Table 2 Labour force participation and cognitive and non-cognitive skills

\begin{tabular}{|c|c|c|c|c|c|c|c|}
\hline & (1) & $(2)$ & (3) & (4) & (5) & (6) & (7) \\
\hline Numeracy & & 0.045 & & & 0.045 & 0.046 & 0.045 \\
\hline Conscientiousness & & & $0.153^{* *}$ & & $0.157^{* * *}$ & & $0.153^{* *}$ \\
\hline Extraversion & & & -0.014 & & -0.011 & & -0.011 \\
\hline Agreeableness & & & $-0.112^{*}$ & & $-0.114^{*}$ & & $-0.113^{*}$ \\
\hline Openness & & & 0.035 & & 0.032 & & 0.033 \\
\hline Neuroticism & & & $-0.098^{* * *}$ & & $-0.097^{* * *}$ & & $-0.094^{* * *}$ \\
\hline Perseverance of effort & & & & 0.012 & & 0.017 & -0.004 \\
\hline Consistency of interest & & & & 0.037 & & 0.033 & 0.014 \\
\hline Observations & 4454 & 4454 & 4454 & 4454 & 4454 & 4454 & 4454 \\
\hline$R^{2}$ & 0.285 & 0.287 & 0.298 & 0.287 & 0.300 & 0.289 & 0.300 \\
\hline
\end{tabular}

${ }^{*} \mathrm{p}<0.05,{ }^{* *} \mathrm{p}<0.01,{ }^{* * *} \mathrm{p}<0.001$. Control variables: age, age square, gender, years of education. Dependent variable, numeracy and non-cognitive skills are standardised. Full estimation results are presented in Additional file 3: Table S2

Agreeableness and Neuroticism are associated with lower levels of education (specification 5). In contrast to previous studies (e.g. Poropat 2009; Van Eijck and de Graaf 2004), we do not find an association between Conscientiousness and educational attainment. However, recent findings by Rammstedt et al. (2017) suggest that this association is actually non-linear. An additional analysis including quadratic terms of personality traits in the model confirms a hump-shaped association between conscientiousness and educational attainment. Individuals with an intermediate level of conscientiousness have the highest average educational attainment. The results for the other Big Five traits support the linear representation of their relationship with education. Only the Consistency of interest facet of Grit is positively correlated with years of education and its effect is half of the size of the effect of Openness, which is the strongest predictor among Big Five traits $(\beta=0.07$ and $\beta=0.14$ respectively).

It has to be stressed that personality traits and cognitive skills are measured at the same point in time for the entire sample. This means that older individuals finished their formal education many years previously while the young are often still in education. Therefore, we can only examine whether personality helps to explain individual variation in educational attainment measured by completed years of schooling, which is a censored measure for the younger cohorts.

As socio-economic status (SES) is believed to be one of the main determinants of educational attainment (Van Eijck and de Graaf 2004), we include controls for parental education to account for it. To provide results comparable to earlier research, appendix reports the results of the analysis without controlling for SES (Table 10). The standardised effects of Big Five traits are around 1.5 times higher than in the model presented below.

\section{Labour market outcomes}

Several studies suggest that personality traits predict labour market outcomes. The decision to participate or not in the labour market appears to be related to individual personality traits. Controlling for education, age and gender, conscientious individuals are more likely to be active on the labour market while agreeable and neurotic individuals are less likely (Table 2 , specification 5 ). The relationship between cognitive skills as measured by 
Table 3 Employability and cognitive and non-cognitive skills

\begin{tabular}{|c|c|c|c|c|c|c|c|}
\hline & (1) & (2) & (3) & (4) & (5) & (6) & (7) \\
\hline Numeracy & & 0.038 & & & 0.040 & 0.045 & 0.046 \\
\hline Conscientiousness & & & 0.094 & & 0.095 & & 0.090 \\
\hline Extraversion & & & 0.010 & & 0.012 & & 0.011 \\
\hline Agreeableness & & & -0.089 & & -0.088 & & -0.084 \\
\hline Openness & & & -0.016 & & -0.019 & & -0.024 \\
\hline Neuroticism & & & 0.018 & & 0.019 & & 0.014 \\
\hline Perseverance of effort & & & & 0.055 & & 0.059 & 0.056 \\
\hline Consistency of interest & & & & -0.061 & & -0.065 & -0.063 \\
\hline Observations & 3356 & 3356 & 3356 & 3356 & 3356 & 3356 & 3356 \\
\hline$R^{2}$ & 0.032 & 0.033 & 0.034 & 0.035 & 0.035 & 0.036 & 0.038 \\
\hline
\end{tabular}

${ }^{*} p<0.05,{ }^{* *} p<0.01,{ }^{* * *} p<0.001$ Control variables: age, age squared, gender, years of education. Dependent variable, numeracy and non-cognitive skills are standardised. Full estimation results are presented in Additional file 3: Table S3

PIAAC numeracy scores and labour force participation is positive but not statistically significant. Adding Big Five traits to the model slightly increases the explained variance by $1.4 \%$ while the effects of numeracy and Grit are much smaller (Table 2, specifications 2-4).

Among individuals active in the labour market, cognitive skills and personality traits are unrelated to employment after controlling for basic socio-demographic factors and explain only marginal additional variance (Table 3, specifications 2-4). This lack of a clear association with employment can be partially explained by the differences in job search behaviours between individuals with internal and external locus of control-a trait linked to Neuroticism. People with internal locus of control tend to search for work more intensively. At the same time, they have higher reservation wages (Caliendo et al. 2015). Therefore, the effect on their employability is ambiguous.

The analysis uses the ILO definitions of employment and activity in the labour market. However, there are differences between ILO employment status and the main activity declared by respondents. The share of employed individuals is 67 and $62 \%$ while the share of unemployed is 5 and 8\%, according to the ILO definition and respondents' declarations, respectively. To test the robustness of our findings, we report the results of the analysis using the self-declared main activity of individuals (Tables 11, 12 in Appendix). The relationship between personality and activity on the labour market is not qualitatively different when the different measure of inactivity is used. When we turn to employability the results differ slightly. The positive association between numeracy and employment is much stronger and significant. Also conscientiousness is positively related to employment in this specification. The source of these differences may be the composition of the group of employed: people who work a limited number of hours declare different activities such as education, housework or even unemployment, thus, the group of employed becomes more homogeneous when a self-declared measure is used.

Once a person is employed, the important question is the quality of their job. The most frequently used indicators of job quality are wages and job satisfaction. The Big Five traits explain only slightly more of the variation in wages than cognitive skills: $1.3 \%$ and $0.8 \%$ respectively while Grit explains almost no additional variance (Table 4, specifications 
Table 4 Wages and cognitive and non-cognitive skills

\begin{tabular}{|c|c|c|c|c|c|c|c|}
\hline & (1) & (2) & (3) & (4) & (5) & (6) & (7) \\
\hline Numeracy & & $0.110^{* *}$ & & & $0.104^{* *}$ & $0.109^{* *}$ & $0.103^{* *}$ \\
\hline Conscientiousness & & & $0.228^{* *}$ & & $0.234^{* *}$ & & $0.267^{* * *}$ \\
\hline Extraversion & & & 0.013 & & 0.015 & & 0.014 \\
\hline Agreeableness & & & $-0.271^{* * *}$ & & $-0.265^{* * *}$ & & $-0.275^{* * *}$ \\
\hline Openness & & & 0.003 & & -0.004 & & -0.009 \\
\hline Neuroticism & & & $-0.077^{* *}$ & & $-0.073^{* *}$ & & $-0.083^{* *}$ \\
\hline Perseverance of effort & & & & -0.023 & & -0.013 & -0.024 \\
\hline Consistency of interest & & & & -0.028 & & -0.034 & -0.045 \\
\hline Observations & 2059 & 2059 & 2059 & 2059 & 2059 & 2059 & 2059 \\
\hline$R^{2}$ & 0.371 & 0.379 & 0.384 & 0.373 & 0.391 & 0.381 & 0.394 \\
\hline
\end{tabular}

${ }^{*} p<0.05,{ }^{* *} p<0.01,{ }^{* * *} p<0.001$ Control variables: age, age squared, gender, years of education, 1-digit ISCO. ISCO $=0$ excluded, top and bottom $1 \%$ of wage distribution excluded. Logarithm of wages. Dependent variable, numeracy and noncognitive skills are standardised. Full estimation results are presented in Additional file 3: Table S4

2-4). Considering Big Five traits and cognitive skills jointly (specification 5) increases the explained variance by $2 \%$. Conscientious individuals are more likely to earn more. By contrast, Agreeableness and Neuroticism are associated with lower wages. These results are in line with empirical studies on Big Five from other countries (e.g. Mueller and Plug 2006; Nyhus and Pons 2005). In contrast to studies conducted in US (Mueller and Plug 2006; O'Connell and Sheikh 2011), Openness is not related to wages in our sample. Additional analysis also did not replicate previous findings on a hump-shaped relationship between openness and wages reported by Rammstedt et al. (2017). The effect sizes of Conscientiousness and Agreeableness on wages are over two times higher than the effect of numeracy $(\beta=0.23, \beta=0.27, \beta=0.10)$. Cognitive and non-cognitive skills might affect wages indirectly via the choice of occupation. When we compare the influence of cognitive and non-cognitive skills on wages without controlling for occupation the difference decreases but the effects of Conscientiousness and Agreeableness are still one and a half times higher. Neither of the facets of Grit is related to wages in our sample.

The next dimension of job quality examined is job satisfaction which is by definition subjective. Results in Table 5 show that personality traits do better at predicting job satisfaction than do cognitive skills. Big Five and Grit traits explain 3.2 and $2 \%$ additional variance respectively. Conscientiousness is linked to higher job satisfaction $(\beta=0.23)$ while Neuroticism is linked to lower job satisfaction $(\beta=-0.12)$. Perseverance of effort is associated with higher levels of job satisfaction $(\beta=0.17)$.

\section{Social outcomes}

In addition to examining the relationships between personality measures and educational and labour market outcomes, it is also useful to examine their relationship with social outcomes. Following OECD $(2007 ; 2013 a)$, interpersonal trust and health are considered as social outcomes.

Big Five and Grit traits explain more additional variance in health than cognitive skills: 2.6, 1.5 and $0.3 \%$ respectively (Table 6, specifications 2-4). Considering Big Five traits and cognitive skills jointly (specification 5 ) increases the explained variance by $2.9 \%$. In line with the earlier research on PIAAC (da Costa et al. 2014; OECD 2013a), numeracy 
Table 5 Job satisfaction and cognitive and non-cognitive skills

\begin{tabular}{|c|c|c|c|c|c|c|c|}
\hline & (1) & (2) & (3) & (4) & (5) & (6) & (7) \\
\hline Numeracy & & -0.001 & & & 0.001 & 0.011 & 0.009 \\
\hline Conscientiousness & & & $0.225^{*}$ & & $0.225^{*}$ & & 0.162 \\
\hline Extraversion & & & 0.107 & & 0.107 & & $0.107^{*}$ \\
\hline Agreeableness & & & -0.157 & & -0.157 & & -0.131 \\
\hline Openness & & & 0.033 & & 0.033 & & 0.031 \\
\hline Neuroticism & & & $-0.116^{* * *}$ & & $-0.116^{* * *}$ & & $-0.107^{* *}$ \\
\hline Perseverance of effort & & & & $0.168^{* * *}$ & & $0.169^{* * *}$ & $0.128^{* * *}$ \\
\hline Consistency of interest & & & & -0.034 & & -0.035 & -0.037 \\
\hline Observations & 2507 & 2507 & 2507 & 2507 & 2507 & 2507 & 2507 \\
\hline$R^{2}$ & 0.021 & 0.021 & 0.053 & 0.041 & 0.053 & 0.041 & 0.063 \\
\hline
\end{tabular}

${ }^{*} p<0.05,{ }^{* *} p<0.01,{ }^{* * *} p<0.001$ Control variables: age, age squared, gender, years of education, 1-digit ISCO. ISCO $=0$ excluded. Dependent variable, numeracy and non-cognitive skills are standardised. Full estimation results are presented in Additional file 3: Table S5

proficiency is positively linked to health but the effect is small $(\beta=0.06)$. Lower levels of Conscientiousness and Extraversion are associated with lower levels of health while lower levels of Neuroticism are associated with higher levels of health. A standard deviation increase in Perseverance of effort results in a 0.10 SD increase in health, holding all other variables constant. Also Consistency of interest is positively associated with health but the effect is not significant.

Also in the case of trust, Big Five and Grit traits explain more additional variance than cognitive skills: $1.3,0.9$ and $0.7 \%$ respectively (Table 7, specifications 2-4). Considering Big Five traits and cognitive skills jointly (specification 5) increases the explained variance in trust by $1.9 \%$. As in the case of health, numeracy is positively correlated with trust and the effect is stronger $(\beta=0.09)$. Neuroticism is the only Big Five trait associated with trust. Neurotic people are more likely to report lower levels of trust and the absolute effect size is similar to numeracy. The results did not replicate findings on the positive association between trust and Openness/Agreeableness and the negative association between trust and Conscientiousness (Dohmen et al. 2008). Perseverance of effort

Table 6 Health and cognitive and non-cognitive skills

\begin{tabular}{|c|c|c|c|c|c|c|c|}
\hline & (1) & $(2)$ & (3) & (4) & (5) & (6) & (7) \\
\hline Numeracy & & $0.057^{*}$ & & & $0.059^{*}$ & $0.064^{* *}$ & $0.063^{* *}$ \\
\hline Conscientiousness & & & $0.123^{*}$ & & $0.129 *$ & & 0.072 \\
\hline Extraversion & & & $0.073^{* *}$ & & $0.076^{* *}$ & & $0.075^{* *}$ \\
\hline Agreeableness & & & -0.042 & & -0.045 & & -0.024 \\
\hline Openness & & & -0.045 & & -0.048 & & -0.046 \\
\hline Neuroticism & & & $-0.158^{* * *}$ & & $-0.156^{* * *}$ & & $-0.145^{* * *}$ \\
\hline Perseverance of effort & & & & $0.090^{* * *}$ & & $0.096^{* * *}$ & $0.086^{* * *}$ \\
\hline Consistency of interest & & & & $0.046^{*}$ & & 0.041 & 0.017 \\
\hline Observations & 4454 & 4454 & 4454 & 4454 & 4454 & 4454 & 4454 \\
\hline$R^{2}$ & 0.308 & 0.311 & 0.334 & 0.323 & 0.337 & 0.326 & 0.345 \\
\hline
\end{tabular}

${ }^{*} p<0.05,{ }^{* *} p<0.01,{ }^{* * *} p<0.001$. Control variables: age, age squared, gender, years of education, employment status. Dependent variable, numeracy and non-cognitive skills are standardised. Full estimation results are presented in Additional file 3: Table S6 
Table 7 Social trust and cognitive and non-cognitive skills

\begin{tabular}{|c|c|c|c|c|c|c|c|}
\hline & (1) & (2) & (3) & (4) & (5) & (6) & (7) \\
\hline Numeracy & & $0.093^{* * *}$ & & & $0.086^{* * *}$ & $0.086^{* * *}$ & $0.083^{* *}$ \\
\hline Conscientiousness & & & -0.089 & & -0.079 & & -0.026 \\
\hline Extraversion & & & 0.050 & & 0.055 & & 0.056 \\
\hline Agreeableness & & & 0.047 & & 0.043 & & 0.023 \\
\hline Openness & & & -0.042 & & -0.047 & & -0.049 \\
\hline Neuroticism & & & $-0.096^{* * *}$ & & $-0.094^{* * *}$ & & $-0.105^{* * *}$ \\
\hline Perseverance of effort & & & & $-0.100^{* * *}$ & & $-0.091^{* * *}$ & $-0.080^{* *}$ \\
\hline Consistency of interest & & & & 0.006 & & -0.000 & -0.017 \\
\hline Observations & 4454 & 4454 & 4454 & 4454 & 4454 & 4454 & 4454 \\
\hline$R^{2}$ & 0.037 & 0.044 & 0.050 & 0.046 & 0.056 & 0.052 & 0.063 \\
\hline
\end{tabular}

${ }^{*} p<0.05,{ }^{* *} p<0.01,{ }^{* * *} p<0.001$. Control variables: age, age squared, gender, years of education, employment status. Dependent variable, numeracy and non-cognitive skills are standardised. Full estimation results are presented in Additional file 3: Table S7

is negatively associated with trust and its effect is similar to the effect of Neuroticism $(\beta=-0.09)$.

\section{Life satisfaction}

General life satisfaction is one of the central outcomes covering different life domains. In contrast to health and trust, life satisfaction is not related to cognitive skills. However, the associations with personality traits are very strong. Big Five traits increase the explained variance in life satisfaction by $5.8 \%$ while Grit does so by $5.2 \%$. For a standard deviation increase in Conscientiousness, life satisfaction increases by 0.27 SD, holding all other variables constant. Also Extraversion has a positive relationship with life satisfaction $(\beta=0.11)$, while Neuroticism has a negative one $(\beta=-0.13)$. Similar to Conscientiousness, both facets of Grit are positively related to life satisfaction but the effect of Perseverance of effort is much stronger than the Consistency of interest $(\beta=0.19$ and $\beta=0.07$ respectively).

Table 8 Life satisfaction and cognitive and non-cognitive skills

\begin{tabular}{|c|c|c|c|c|c|c|c|}
\hline & (1) & (2) & (3) & (4) & (5) & (6) & (7) \\
\hline Numeracy & & -0.002 & & & 0.011 & 0.010 & 0.016 \\
\hline Conscientiousness & & & $0.271^{* * *}$ & & $0.272^{* * *}$ & & $0.169^{* *}$ \\
\hline Extraversion & & & $0.112^{* *}$ & & $0.113^{* *}$ & & $0.111^{* *}$ \\
\hline Agreeableness & & & -0.111 & & -0.111 & & -0.073 \\
\hline Openness & & & -0.001 & & -0.001 & & 0.003 \\
\hline Neuroticism & & & $-0.131^{* * *}$ & & $-0.131^{* * *}$ & & $-0.108^{* * *}$ \\
\hline Perseverance of effort & & & & $0.190^{* * *}$ & & $0.191^{* * *}$ & $0.137^{* * *}$ \\
\hline Consistency of interest & & & & $0.067^{*}$ & & $0.066^{*}$ & 0.055 \\
\hline Observations & 4454 & 4454 & 4454 & 4454 & 4454 & 4454 & 4454 \\
\hline$R^{2}$ & 0.071 & 0.071 & 0.129 & 0.124 & 0.129 & 0.124 & 0.155 \\
\hline
\end{tabular}

${ }^{*} p<0.05,{ }^{* *} p<0.01,{ }^{* * *} p<0.001$. Control variables: age, age squared, gender, years of education, employment status. Dependent variable, numeracy and non-cognitive skills are standardised. Full estimation results are presented in Additional file 3: Table S8 


\section{The incremental validity of Grit}

The construct of Grit is often related conceptually to the Big Five factor of Conscientiousness and the empirical correlations between the two are high (see Introduction). An important question, therefore, is whether Grit provides some extra information when we control for the Big Five traits. The correlation between Grit and Conscientiousness in the postPIAAC sample is 0.37 , indicating that $14 \%$ of variation in scores across these scales is shared.

Not all associations between the outcomes and Grit subscales and Conscientiousness follow a similar pattern (specification 7, Tables 1, 2, 3, 4, 5, 6, 7, 8). Some outcomes are related to one of the Grit subscales but not to Conscientiousness (educational attainment and trust), some are associated with Conscientiousness but not with Grit (labour force participation, wages) and for some we observe similar effects (job satisfaction, health and life satisfaction). The facets of Grit explain around $1 \%$ of additional variance in educational attainment, job satisfaction, health and trust, even after adjusting for the effects of cognitive skills and Big Five traits. Moreover, they explain $2.6 \%$ of additional variance in life satisfaction. Taking into account that for some outcomes the overall variance explained in the full model is even as low as $6 \%$ (in the case of trust), these additional effects are not negligible. ${ }^{8}$

In sum, Grit has an incremental value for some of the life outcomes examined when adjusting for the effects of numeracy and Big Five personality traits. To date, its predictive power was mainly validated in the context of school outcomes: attainment and grades. In this respect, and in line with previous research (Duckworth and Quinn 2009; Eskreis-Winkler et al. 2014), Grit is significantly associated with educational attainment after controlling for Big Five traits and explains an additional $1 \%$ of variance while Conscientiousness is not related to education. However, this effect is driven by the Consistency of interest facet of Grit, and not Perseverance of effort as suggested by Credé et al. (2017). Also, Grit is significantly and negatively associated with trust and improves the model while Conscientiousness does not. Regarding health, life satisfaction and to lesser extent job satisfaction, much of the effect of Grit in explaining these outcomes is shared with Conscientiousness. In contrast to previous studies (e.g. EskreisWinkler et al. 2014; Suzuki et al. 2015), we do not find a positive relationship between Grit and economic outcomes measured by labour force participation, employability and wages.

\section{Discussion}

The present study seeks to assess the analytical importance of personality measures compared to cognitive skills measured in PIAAC and to compare the explanatory power of the Big Five and Grit constructs in a large representative sample of adults in Poland. It investigates the relationships between personality and a wide range of important life outcomes, as well as the incremental validity of Grit when Big Five traits are accounted for.

Overall, the results confirm earlier findings from the literature that differences in personality traits are important in explaining differences in life outcomes. For most of the subjective outcomes, the Big Five traits outperform cognitive skills in predictive power. Only educational attainment is more strongly related to cognitive skills, while for wages, the explanatory power of personality and cognitive skills is similar. After controlling for sociodemographic characteristics and cognitive skills, Big Five traits are incrementally predictive of all life outcomes except

\footnotetext{
${ }^{8}$ For all the outcomes when analysing Grit as an overall score from the unidimensional model, the variance explained is the same or slightly lower ( $\max . \Delta \mathrm{R}^{2}=0.004$ ) than in the models analysing the facets of Grit from the two-factor model (Appendix: Table 13).
} 
for employability. The strongest effects are observed for subjective, self-declared outcomes such as health or job satisfaction. The stronger predictive power of Big Five for subjective outcomes confirms the results of Rammstedt et al. (2017), who argued that it is the same aggregation level of personality measures and the subjective measures which may drive the correlation. Moreover, these higher correlations may be due to the response style on attitudinal and self-evaluative items of Likert scales. Further research on this issue is needed.

The effects of particular traits are largely in agreement with predictions. They confirm similar effects observed in previous studies conducted in the United States and Western Europe. Conscientiousness is positively related to most of the outcomes analysed while Neuroticism has a negative relationship. Extraverted individuals are more likely to attain lower levels of education. They are also more satisfied with their life and job and feel healthier. Agreeableness is associated with lower levels of education and negative labour market outcomes. Openness is strongly and positively related to educational attainment. In contrast to previous studies (e.g. Mueller and Plug 2006; Rammstedt et al. 2017) we do not find relationship between Openness and wages.

Moving beyond the research focusing on the Big Five model, we complement the few studies that have examined the incremental validity of Grit (e.g. Duckworth and Quinn 2009; EskreisWinkler et al. 2014; Suzuki et al. 2015) and extend it across more life domains. Using a representative sample of adults, we show that the Grit-S scale has less predictive power than the BFI-S, but jointly considering both scales increases the explained variance in many outcomes. The effects on many outcomes are not smaller than the effects of Big Five traits. Although literature suggests that Grit and Conscientiousness are conceptually similar (e.g. Credé et al. 2017), they share the effects on outcomes only in case of health, life satisfaction and to a lesser extent job satisfaction. In contrast to Conscientiousness, Grit is unrelated to labour market outcomes but explains additional variation in educational attainment and trust. These results suggest that the emerging view of Grit as a facet of Conscientiousness is rather premature.

Further, we have shown that the facets of Grit do not predict life outcomes equally well. The associations of Grit with all the subjective outcomes are driven mainly by the Perseverance of effort facet, while it is the Consistency of interest facet that is related to educational attainment. This last result contradicts the findings of Credé et al. (2017) who showed that the Perseverance facet exhibits much stronger relations with academic performance. The reason for this difference could be the nature of the educational outcomes analysed. While Credé et al. (2017) rely on grades, we look at the highest completed level of education. Changing one's interests often may not harm one's grades, analysed as GPA counted across subjects, but it may make it harder to complete a higher educational level.

This study is not without limitations. First, the cognitive skills were assessed around three years earlier than personality traits and life outcomes which may result in weaker associations than in reality. However, there is longitudinal evidence that cognitive skills analysed are relatively stable over such a period or even fixed early in life (Desjardins and Warnke 2012). Hill et al. (2008) show that for school children, average annual gains in literacy and numeracy decline with age and are already marginal by the age of 17 . Second, although the results presented describe relationships between personality traits and life outcomes, drawing any conclusions about causality is not possible with the available data. Bidirectional influences are likely to underlie the observed cross-sectional associations. This is especially true for the analysis of educational attainment, as many respondents completed 
their education many years before the personality traits were assessed and there is also evidence that personality can be shaped by the educational system (Dahmann and Anger 2014) and tertiary education (Kassenboehmer et al. 2018). We are aware that the lack of a causal model is a serious limitation for designing policy interventions and for policy analysis. Nevertheless, the analysis is of an exploratory nature and results stress the importance of the topic, which might motivate further work on improving our understanding of the mechanisms linking personality traits and life outcomes. Some authors show that personality can change during one's lifespan. Specht et al. (2011) conclude that the relationship of personality with age is complex and curvilinear and can change due to the major life events. To better understand to what degree personality may change over the lifespan and what factors affect such changes we need more longitudinal studies on personality. Finally, the relationships between personality traits and life outcomes are dynamic and complex. Personality may influence life outcomes not only directly but also indirectly by contributing to the development of cognitive skills and to the completion of educational qualifications. Moreover, the analysed outcomes are also related with each other. We partially account for indirect effects by controlling for educational attainment in all the models and for the employment status in the social outcomes and life satisfaction models. However, examining these relationships in one general model might be an avenue of future research.

The nature of personality traits differs from that of cognitive skills, such that "more" does not necessarily mean "better". From the policy-making perspective, the decision which traits to foster may therefore be a difficult one. The value of incorporating personality traits measures into international large-scale assessments would lie in the possibility to compare the traits-outcome relationships between countries and groups within countries, not in national rankings on personality traits. Some personality traits are beneficial in some specific work environments but not in others. For example, Agreeableness is on average related to lower wages but improves teamwork (Tasa et al. 2011). A potential solution would be to promote beneficial behaviours in specific contexts, e.g. being disagreeable when negotiating wages but agreeable when working on a project involving teamwork.

\section{Conclusion}

In sum, given the potential benefits and relatively small burden on respondents in terms of required time it seems advisable to incorporate measures of personality traits into competence surveys as they contribute to explaining the variability in policy-relevant outcomes. Taking into account the length of the scales analysed (eight and fifteen items for Grit-S and BFI-S respectively), using the Big Five model seems preferable to using Grit when a broad range of life outcomes is of interest, as the former covers multiple aspects of personality. However, using both scales offers an improvement in explanatory power.

\section{Additional files}

Additional file 1. Literacy—-full estimation results (OLS) Tables S1-S8.

Additional file 2. Numeracy-non-linear models Tables S1-S6.

Additional file 3. Numeracy_full estimation results (OLS) Tables S1-S8. 
Author details

'Educational Research Institute, Warsaw, Poland. ${ }^{2}$ Warsaw School of Economics, Warsaw, Poland.

\section{Acknowledgements}

We thank Artur Pokropek, Michał Sitek, William Thorn and three anonymous referees for their insightful comments and suggestions. We gratefully acknowledge financial support from the Organisation for Economic Co-operation and Development (OECD). All errors are ours.

Competing interests

The authors declare that they have no competing interests.

Availability of data and materials

The postPIAAC dataset is administered by the Educational Research Institute in Warsaw and is not publicly available online.

Ethics approval and consent to participate

Not applicable.

Funding

We thank the Organisation for Economic Co-operation and Development (OECD) for funding the analysis.

\section{Appendix}

See Tables 9, 10, 11, 12 and 13.

Table 9 Summary statistics of life outcomes

\begin{tabular}{llccll}
\hline Variable & N & Mean & SD & Min & Max \\
\hline Years of education & 4454 & 13.19 & 2.86 & 6 & 21 \\
LM participation & 4454 & 0.72 & 0.45 & 0 & 1 \\
Employment & 3356 & 0.93 & 0.25 & 0 & 1 \\
Job satisfaction & 2507 & 1.99 & 0.65 & 1 & 3 \\
Hourly wages (PLN) & 2059 & 17.18 & 10.30 & 4.9 & 83.3 \\
Health & 4454 & 3.15 & 0.89 & 1 & 5 \\
Trust & 4454 & 2.85 & 1.37 & 1 & 6 \\
Life satisfaction & 4454 & 3.63 & 1.03 & 1 & 5 \\
\hline
\end{tabular}

Table 10 Educational attainment and cognitive and non-cognitive skills (without controlling for SES)

\begin{tabular}{|c|c|c|c|c|c|c|c|}
\hline & (1) & $(2)$ & (3) & (4) & (5) & (6) & (7) \\
\hline Numeracy & & $0.440^{* * *}$ & & & $0.419^{* * *}$ & $0.434^{* * *}$ & $0.413^{* * *}$ \\
\hline Conscientiousness & & & $-0.122^{*}$ & & -0.058 & & $-0.106^{*}$ \\
\hline Extraversion & & & $-0.138^{* * *}$ & & $-0.088^{* * *}$ & & $-0.086^{* *}$ \\
\hline Agreeableness & & & -0.089 & & -0.090 & & -0.071 \\
\hline Openness & & & $0.310^{* * *}$ & & $0.230^{* * *}$ & & $0.232^{* * *}$ \\
\hline Neuroticism & & & $-0.073^{* * *}$ & & $-0.048^{*}$ & & -0.030 \\
\hline Perseverance of effort & & & & -0.042 & & 0.009 & 0.025 \\
\hline Consistency of interest & & & & $0.137^{* * *}$ & & $0.078^{* * *}$ & $0.080^{* * *}$ \\
\hline Age & $0.067^{* * *}$ & $0.058^{* * *}$ & $0.070^{* * *}$ & $0.061^{* * *}$ & $0.060^{* * *}$ & $0.054^{* * *}$ & $0.058^{* * *}$ \\
\hline Age \# age & $-0.001^{* * *}$ & $-0.001^{* * *}$ & $-0.001^{* * *}$ & $-0.001^{* * *}$ & $-0.001^{* * *}$ & $-0.001^{* * *}$ & $-0.001^{* * *}$ \\
\hline Female & $0.300^{* * *}$ & $0.308^{* * *}$ & $0.356^{* * *}$ & $0.300^{* * *}$ & $0.347^{* * *}$ & $0.302^{* * *}$ & $0.336^{* * *}$ \\
\hline Constant & $-1.124^{* * *}$ & $-1.090^{* * *}$ & $-1.277^{* * *}$ & $-1.010^{* * *}$ & $-1.186^{* * *}$ & $-0.996^{* * *}$ & $-1.131^{* * *}$ \\
\hline Observations & 4454 & 4454 & 4454 & 4454 & 4454 & 4454 & 4454 \\
\hline$R^{2}$ & 0.086 & 0.271 & 0.128 & 0.100 & 0.292 & 0.278 & 0.300 \\
\hline
\end{tabular}

${ }^{*} \mathrm{p}<0.05,{ }^{* *} \mathrm{p}<0.01,{ }^{* * *} \mathrm{p}<0.001$. Dependent variable, numeracy and non-cognitive skills are standardised 
Table 11 Self-declared labour force participation and cognitive and non-cognitive skills

\begin{tabular}{|c|c|c|c|c|c|c|c|}
\hline & (1) & (2) & (3) & (4) & (5) & (6) & (7) \\
\hline Numeracy & & 0.005 & & & 0.005 & 0.008 & 0.007 \\
\hline Conscientiousness & & & $0.119^{*}$ & & $0.119^{*}$ & & $0.107^{*}$ \\
\hline Extraversion & & & 0.007 & & 0.008 & & 0.007 \\
\hline Agreeableness & & & -0.084 & & -0.084 & & -0.080 \\
\hline Openness & & & 0.013 & & 0.013 & & 0.013 \\
\hline Neuroticism & & & $-0.076^{* * *}$ & & $-0.076^{* * *}$ & & $-0.075^{* * *}$ \\
\hline Perseverance of effort & & & & 0.040 & & 0.041 & 0.028 \\
\hline Consistency of interest & & & & 0.005 & & 0.004 & -0.010 \\
\hline Age & $0.189^{* * *}$ & $0.189^{* * *}$ & $0.184^{* * *}$ & $0.187^{* * *}$ & $0.184^{* * *}$ & $0.188^{* * *}$ & $0.184^{* * *}$ \\
\hline Age \# age & $-0.002^{* * *}$ & $-0.002^{* * *}$ & $-0.002^{* * *}$ & $-0.002^{* * *}$ & $-0.002^{* * *}$ & $-0.002^{* * *}$ & $-0.002^{* * *}$ \\
\hline Female & $-0.383^{* * *}$ & $-0.382^{* * *}$ & $-0.360^{* * *}$ & $-0.390^{* * *}$ & $-0.359^{* * *}$ & $-0.389^{* * *}$ & $-0.363^{* * *}$ \\
\hline Years of education & $0.049^{* * *}$ & $0.048^{* * *}$ & $0.047^{* * *}$ & $0.048^{* * *}$ & $0.046^{* * *}$ & $0.047^{* * *}$ & $0.046^{* * *}$ \\
\hline Constant & $-3.750^{* * *}$ & $-3.742^{* * *}$ & $-3.634^{* * *}$ & $-3.701^{* * *}$ & $-3.625^{* * *}$ & $-3.688^{* * *}$ & $-3.620^{* * *}$ \\
\hline Observations & 4452 & 4452 & 4452 & 4452 & 4452 & 4452 & 4452 \\
\hline$R^{2}$ & 0.301 & 0.301 & 0.309 & 0.303 & 0.309 & 0.303 & 0.309 \\
\hline
\end{tabular}

${ }^{*} \mathrm{p}<0.05,{ }^{* *} \mathrm{p}<0.01,{ }^{* * *} \mathrm{p}<0.001$. Dependent variable, numeracy and non-cognitive skills are standardised

Table 12 Self-declared employability and cognitive and non-cognitive skills

\begin{tabular}{|c|c|c|c|c|c|c|c|}
\hline & (1) & (2) & (3) & (4) & $(5)$ & (6) & (7) \\
\hline Numeracy & & $0.090^{* *}$ & & & $0.090^{* *}$ & $0.093^{* *}$ & $0.092^{* *}$ \\
\hline Conscientiousness & & & $0.143^{*}$ & & $0.146^{*}$ & & $0.135^{*}$ \\
\hline Extraversion & & & -0.018 & & -0.012 & & -0.012 \\
\hline Agreeableness & & & -0.123 & & -0.122 & & -0.117 \\
\hline Openness & & & -0.019 & & -0.025 & & -0.028 \\
\hline Neuroticism & & & -0.021 & & -0.018 & & -0.019 \\
\hline Perseverance of effort & & & & 0.028 & & 0.036 & 0.036 \\
\hline Consistency of interest & & & & -0.005 & & -0.013 & -0.021 \\
\hline Age & $0.045^{* * *}$ & $0.045^{* * *}$ & $0.041^{* *}$ & $0.045^{* * *}$ & $0.041^{* *}$ & $0.045^{* * *}$ & $0.042^{* *}$ \\
\hline Age \# age & $-0.001^{* * *}$ & $-0.001^{* * *}$ & $-0.000^{* *}$ & $-0.001^{* * *}$ & $-0.000^{* *}$ & $-0.001^{* * *}$ & $-0.000^{* *}$ \\
\hline Female & $-0.117^{* *}$ & $-0.095^{*}$ & $-0.108^{*}$ & $-0.122^{* *}$ & -0.089 & $-0.101^{*}$ & $-0.091^{*}$ \\
\hline Years of education & $0.066^{* * *}$ & $0.051^{* * *}$ & $0.068^{* * *}$ & $0.066^{* * *}$ & $0.053^{* * *}$ & $0.051^{* * *}$ & $0.053^{* * *}$ \\
\hline Constant & $-1.744^{* * *}$ & $-1.557^{* * *}$ & $-1.697^{* * *}$ & $-1.731^{* * *}$ & $-1.516^{* * *}$ & $-1.543^{* * *}$ & $-1.518^{* * *}$ \\
\hline Observations & 3224 & 3224 & 3224 & 3224 & 3224 & 3224 & 3224 \\
\hline$R^{2}$ & 0.042 & 0.048 & 0.046 & 0.043 & 0.052 & 0.049 & 0.052 \\
\hline
\end{tabular}

${ }^{*} \mathrm{p}<0.05,{ }^{* *} \mathrm{p}<0.01,{ }^{* * *} \mathrm{p}<0.001$. Dependent variable, numeracy and non-cognitive skills are standardised 
Table 13 Comparison of the unidimensional and the two-factor Grit models

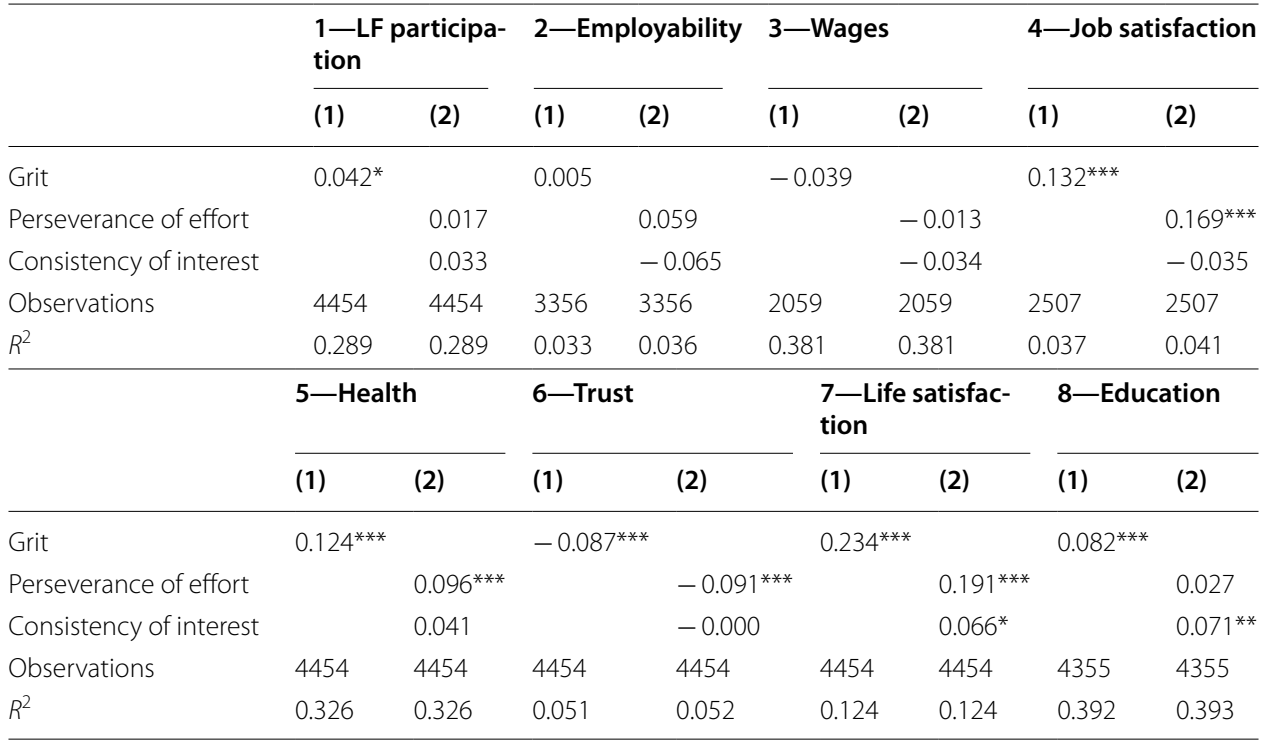

${ }^{*} p<0.05,{ }^{* *} p<0.01,{ }^{* * *} p<0.001$. (1)-The unidimensional model of Grit. (2)-two-factor model of Grit. Control variables: age, age squared, gender, years of education (outcomes 1-7), 1-digit ISCO (outcomes 3, 4), employment status (outcomes 5-7), parents education (outcome 8). Dependent variables, numeracy and non-cognitive skills are standardised

\section{Publisher's Note}

Springer Nature remains neutral with regard to jurisdictional claims in published maps and institutional affiliations.

Received: 22 December 2017 Accepted: 20 April 2018

Published online: 30 April 2018

\section{References}

Almlund, M., Duckworth, A. L., Heckman, J., Kautz, T. (2011). Personality psychology and economics. In Handbook of economics of education (Vol. 4). New York: Elsevier.

Anderson, M. R. (2010). Community psychology, political efficacy, and trust. Political Psychology, 31(1), $59-84$.

Barrick, M. R., \& Mount, M. K. (1991). The Big Five personality dimensions and job performance: A meta-analysis. Personnel Psychology, 44(1), 1-26.

Bazelais, P., Lemay, D. J., \& Doleck, T. (2016). How does Grit impact college students' academic achievement in science? European Journal of Science and Mathematics Education, 4(1), 33-43.

Bertling, J. (2016). New Survey Questionnaire Indicators in PISA and NAEP. Presented at the RWJF \& ETS Workshop, Princeton. Retrieved July, 2017 from https://www.ets.org/s/achievement_gap/conferences/rwjf/presentations.html.

Borghans, L., Duckworth, A. L., Heckman, J. J., \& Ter Weel, B. (2008). The economics and psychology of personality traits. Journal of Human Resources, 43(4), 972-1059.

Caliendo, M., Cobb-Clark, D. A., \& Uhlendorff, A. (2015). Locus of control and job search strategies. Review of Economics and Statistics, 97(1), 88-103.

Costa, P. T., Jr., \& McCrae, R. R. (1985). The NEO Personality Inventory manual. Odessa: Psychological Assessment Resources.

Credé, M., Tynan, M. C., \& Harms, P. D. (2017). Much ado about Grit: A meta-analytic synthesis of the Grit literature. Journal of Personality and Social Psychology, 113(3), 492

Cunningham, W., Torrado, M. P., Sarzosa, M. (2016). Cognitive and non-cognitive skills for the Peruvian labor market: Addressing measurement error through latent skills estimations. World Bank Policy Research Working Paper, (7550),

da Costa, P. D., Rodrigues, M., Vera-Toscano, E., \&Weber, A. (2014). Education, adult skills and social outcomes: Empirical evidence from the survey on adult skills (PIAAC 2013). Luxembourg: Publications Office of the European Union.

Dahmann, S., Anger, S. (2014). The impact of education on personality: Evidence from a German high school reform. IZA Discussion Paper, (8139).

Datu, J. A. D., Valdez, J. P. M., \& King, R. B. (2016). Perseverance counts but consistency does not! Validating the short Grit scale in a collectivist setting. Current Psychology, 35(1), 121-130. https://doi.org/10.1007/s12144-015-9374-2.

DeNeve, K. M., \& Cooper, H. (1998). The happy personality: A meta-analysis of 137 personality traits and subjective wellbeing. Psychological Bulletin, 124(2), 197.

Desjardins, R., Warnke, A. J. (2012). Ageing and skills: A review and analysis of skill gain and skill loss over the lifespan and over time. OECD Education Working Papers, (72).

DiStefano, C., \& Motl, R. W. (2006). Further investigating method effects associated with negatively worded items on selfreport surveys. Structural Equation Modeling, 13(3), 440-464. 
Dohmen, T., Falk, A., Huffman, D., \& Sunde, U. (2008). Representative trust and reciprocity: Prevalence and determinants. Economic Inquiry, 46(1), 84-90.

Drago, F. (2011). Self-esteem and earnings. Journal of Economic Psychology, 32(3), 480-488.

Duckworth, A. L., Kirby, T. A., Tsukayama, E., Berstein, H., \& Ericsson, K. A. (2011). Deliberate practice spells success: Why grittier competitors triumph at the national spelling bee. Social Psychological and Personality Science, 2(2), 174-181.

Duckworth, A. L., Peterson, C., Matthews, M. D., \& Kelly, D. R. (2007). Grit: Perseverance and passion for long-term goals. Journal of Personality and Social Psychology, 92(6), 1087-1101. https://doi.org/10.1037/0022-3514.92.6.1087.

Duckworth, A. L., \& Quinn, P. D. (2009). Development and validation of the short Grit scale (GRIT-S). Journal of Personality Assessment, 91(2), 166-174.

Dumfart, B., \& Neubauer, A. C. (2016). Conscientiousness is the most powerful noncognitive predictor of school achievement in adolescents. Journal of Individual Differences, 37(1), 8-15. https://doi.org/10.1027/1614-0001/a000182.

Eskreis-Winkler, L., Shulman, E. P., Beal, S. A., \& Duckworth, A. L. (2014). The Grit effect: Predicting retention in the military, the workplace, school and marriage. Frontiers in Psychology. https://doi.org/10.3389/fpsyg.2014.00036.

Freitag, M., \& Bauer, P. C. (2016). Personality traits and the propensity to trust friends and strangers. The Social Science Journal, 53(4), 467-476. https://doi.org/10.1016/j.soscij.2015.12.002.

Gerlitz, J.-Y., \& Schupp, J. (2005). Zur Erhebung der big-five-basierten persoenlichkeitsmerkmale im SOEP. DIW Research Notes, 4, 2005

Goldberg, L. R., Sweeney, D., Merenda, P. F., \& Hughes, J. E. (1998). Demographic variables and personality: The effects of gender, age, education, and ethnic/racial status on self-descriptions of personality attributes. Personality and Individual Differences, 24(3), 393-403.

Grijalva, E., \& Newman, D. A. (2015). Narcissism and counterproductive work behavior (CWB): Meta-analysis and consideration of collectivist culture, Big Five personality, and narcissism's facet structure. Applied Psychology, 64(1), 93-126.

Hampson, S. E., Goldberg, L. R., Vogt, T. M., \& Dubanoski, J. P. (2007). Mechanisms by which childhood personality traits influence adult health status: Educational attainment and healthy behaviors. Health Psychology, 26(1), 121.

Heckman, J. J., \& Kautz, T. (2012). Hard evidence on soft skills. Labour Economics, 19(4), 451-464.

Heckman, J., Moon, S. H., Pinto, R., Savelyev, P. A., \& Yavitz, A. (2010a). The rate of return to the HighScope Perry Preschool Program. Journal of Public Economics, 94(1), 114-128.

Heckman, J., Moon, S. H., Pinto, R., Savelyev, P., \& Yavitz, A. (2010b). Analyzing social experiments as implemented: A reexamination of the evidence from the HighScope Perry Preschool Program. Quantitative Economics, 1(1), 1-46.

Heckman, J. J., Pinto, R., \& Savelyev, P. A. (2013). Understanding the mechanisms through which an influential early childhood program boosted adult outcomes. The American Economic Review, 103(6), 1-35.

Heineck, G., \& Anger, S. (2010). The returns to cognitive abilities and personality traits in Germany. Labour Economics, 17(3), 535-546.

Henrich, J., Heine, S. J., \& Norenzayan, A. (2010). Most people are not WEIRD. Nature, 466(7302), 29.

Hill, C. J., Bloom, H. S., Black, A. R., \& Lipsey, M. W. (2008). Empirical benchmarks for interpreting effect sizes in research. Child Development Perspectives, 2(3), 172-177.

Hofstede, G. (1980). Culture's consequences: International differences in work-related values. New York: Sage.

Inglehart, R. (2006). Mapping global values. Comparative Sociology, 5(2), 115-136.

Ivcevic, Z., \& Brackett, M. (2014). Predicting school success: Comparing conscientiousness, Grit, and emotion regulation ability. Journal of Research in Personality, 52, 29-36. https://doi.org/10.1016/j.jp.2014.06.005.

John, O. P., Donahue, E. M., \& Kentle, R. L. (1991). The Big Five inventory-versions 4a and 54. Berkeley: University of California, Institute of Personality and Social Research.

John, O. P., \& Srivastava, S. (1999). The Big Five trait taxonomy: History, measurement, and theoretical perspectives. Handbook of Personality Theory and Research, 2(1999), 102-138.

Judge, T. A., Heller, D., \& Mount, M. K. (2002). Five-factor model of personality and job satisfaction: A meta-analysis. Journal of Applied Psychology, 87(3), 530-541.

Kassenboehmer, S. C., Leung, F., \& Schurer, S. (2018). University education and non-cognitive skill development. Oxford Economic Papers, 70(2), 538-562.

Lucas, G. M., Gratch, J., Cheng, L., \& Marsella, S. (2015). When the going gets tough: Grit predicts costly perseverance. Journal of Research in Personality, 59, 15-22. https://doi.org/10.1016/j.jrp.2015.08.004.

McCrae, R. R., \& Costa, P. T., Jr. (1999). A five-factor theory of personality. Handbook of Personality Theory and Research, 2, 139-153.

Mendolia, S., Walker, I. (2014). Do NEETs need Grit? IZA discussion paper No. 8740.

Midkiff, B., Langer, M., Demetriou, C., \& Panter, A. T. (2017). Measuring Grit among first-generation college students: A psychometric analysis. In L. A. van der Ark, M. Wiberg, S. A. Culpepper, J. A. Douglas, \& W.-C. Wang (Eds.), Quantitative psychology (Vol. 196, pp. 407-420). Cham: Springer. https://doi.org/10.1007/978-3-319-56294-0_35.

Mood, C. (2010). Logistic regression: Why we cannot do what we think we can do, and what we can do about it. European Sociological Review, 26(1), 67-82.

Mueller, G., \& Plug, E. (2006). Estimating the effect of personality on male and female earnings. Industrial and Labor Relations Review, 60(1), 3-22. https://doi.org/10.1177/001979390606000101.

Nyhus, E. K., \& Pons, E. (2005). The effects of personality on earnings. Journal of Economic Psychology, 26(3), 363-384. https://doi.org/10.1016/j.joep.2004.07.001.

O'Connell, M., \& Sheikh, H. (2011).'Big Five' personality dimensions and social attainment: Evidence from beyond the campus. Personality and Individual Differences, 50(6), 828-833.

OECD. (2007). Understanding the social outcomes of learning. Paris: OECD Publishing.

OECD. (2013a). OECD skills outlook 2013: First results from the survey of adult skills. Paris: OECD Publishing.

OECD. (2013b). Technical Report of the Survey of Adult Skills (PIAAC). OECD.

OECD. (2016). Skills Matter: Further Results from the Survey of Adult Skills. Paris: OECD Publishing. https://doi. org/10.1787/9789264258051-en

Palczyńska, M., Świst, K. (2016). Measurement properties of non-cognitive scales in the Polish follow-up study on PIAAC (POSTPIAAC). OECD Education Working Paper, (149). 
Poropat, A. E. (2009). A meta-analysis of the five-factor model of personality and academic performance. Psychological Bulletin, 135(2), 322-338.

Prevoo, T., \& ter Weel, B. (2015). The importance of early conscientiousness for socio-economic outcomes: Evidence from the British Cohort Study. Oxford Economic Papers, 67(4), 918-948.

Rammstedt, B., Danner, D., \& Lechner, C. (2017). Personality, competencies, and life outcomes: Results from the German PIAAC longitudinal study. Large-Scale Assessments in Education, 5(1), 2.

Rimfeld, K., Kovas, Y., Dale, P. S., \& Plomin, R. (2016). True Grit and genetics: Predicting academic achievement from personality. Journal of Personality and Social Psychology, 111(5), 780-789. https://doi.org/10.1037/pspp0000089.

Roberts, B. W., Kuncel, N. R., Shiner, R., Caspi, A., \& Goldberg, L. R. (2007). The power of personality: The comparative validity of personality traits, socioeconomic status, and cognitive ability for predicting important life outcomes. Perspectives on Psychological Science, 2(4), 313-345

Specht, J., Egloff, B., \& Schmukle, S. C. (2011). Stability and change of personality across the life course: The impact of age and major life events on mean-level and rank-order stability of the Big Five. Journal of Personality and Social Psychology, 101(4), 862-882.

Suzuki, Y., Tamesue, D., Asahi, K., \& Ishikawa, Y. (2015). Grit and work engagement: A cross-sectional study. PLoS ONE, 10(9), e0137501. https://doi.org/10.1371/journal.pone.0137501.

Tasa, K., Sears, G. J., \& Schat, A. C. H. (2011). Personality and teamwork behavior in context: The cross-level moderating role of collective efficacy. Journal of Organizational Behavior, 32(1), 65-85. https://doi.org/10.1002/job.680.

Van Eijck, K., \& de Graaf, P. M. (2004). The Big Five at school: The impact of personality on educational attainment. The Netherlands Journal of Social Sciences, 40(1), 24-40.

World Bank. (2014). STEP skills measurement: Snapshot 2014. Washington, D.C.: World Bank.

\section{Submit your manuscript to a SpringerOpen ${ }^{\circ}$} journal and benefit from:

- Convenient online submission

- Rigorous peer review

- Open access: articles freely available online

- High visibility within the field

- Retaining the copyright to your article

Submit your next manuscript at $\gg$ springeropen.com 